\title{
Observational properties of a Type Ib Supernova MASTER OT J120451.50+265946.6 in NGC 4080
}

\author{
Mridweeka Singh ${ }^{1,2 \star}$, Kuntal Misra ${ }^{1,3}$, D.K.Sahu ${ }^{4}$, Raya Dastidar ${ }^{1,5}$, \\ Anjasha Gangopadhyay ${ }^{1,2}$, Shubham Srivastav 6 , G. C. Anupama ${ }^{4}$, \\ Subhash Bose ${ }^{7}$, Vladimir Lipunov ${ }^{8,9}$, N. K. Chakradhari ${ }^{2}$, Brajesh Kumar ${ }^{4}$, \\ Brijesh Kumar $^{1}$, S. B. Pandey ${ }^{1}$, Evgeny Gorbovskoy ${ }^{9}$, Pavel Balanutsa ${ }^{9}$ \\ ${ }^{1}$ Aryabhatta Research Institute of observational sciencES, Manora Peak, Nainital 263 001, India \\ ${ }^{2}$ School of Studies in Physics and Astrophysics, Pandit Ravishankar Shukla University, Chattisgarh 492 010, India \\ ${ }^{3}$ Department of Physics, University of California, 1 Shields Ave, Davis, CA 95616-5270, USA \\ ${ }^{4}$ Indian Institute of Astrophysics, Koramangala, Bangalore 560 034, India \\ ${ }^{5}$ Department of Physics 83 Astrophysics, University of Delhi, Delhi 110 007, India \\ ${ }^{6}$ Department of physics, Indian Institute of Technology, Powai, Mumbai 400076, India \\ ${ }^{7}$ Kavli Institute for Astronomy and Astrophysics, Peking University, 5 Yiheyuan Road, Haidian District, Beijing 100871, China \\ ${ }^{8}$ Lomonosov Moscow State University, Physics Department, 119991, Vorobievy hills, 1, Moscow, Russia \\ ${ }^{9}$ Lomonosov Moscow State University, SAI, 119234, Universitetsky pr., 13, Moscow, Russia
}

Accepted XXX. Received YYY; in original form ZZZ

\begin{abstract}
MASTER OT J120451.50+265946.6 (M12045), discovered by the MASTER Global Robotic Net, is a Type Ib supernova (SN) that exploded in NGC 4080. We present the $B V R I$ photometric and spectroscopic observations upto $\sim 250$ days since $B_{\max }$. At the time of discovery the SN was a few weeks past maximum light and our observations capture the linearly declining light curve phase. M12045 declines faster as compared to $\mathrm{SNe} 1999 \mathrm{dn}$ and $2009 \mathrm{jf}$ at comparable epochs. Rigorous spectroscopic monitoring reveals that M12045 is a normal Type Ib SN. The analysis of the nebular phase spectra indicates that $\sim 0.90 \mathrm{M}_{\odot}$ of $\mathrm{O}$ is ejected in the explosion. The line ratio of $[\mathrm{OI}]$ and [CaII] in the nebular phase supports a massive WR progenitor with main sequence mass of $\sim 20 \mathrm{M}_{\odot}$.
\end{abstract}

Key words: supernovae: general - supernovae: individual: MASTER OT J120451.50+265946.6 (M12045) - galaxies: individual: NGC 4080 - techniques: photometric - techniques: spectroscopic

\section{INTRODUCTION}

Type Ib supernovae ( $\mathrm{SNe}$ ) are a subclass of hydrogen deficient $\mathrm{SNe}$ with prominent He features in their early time spectra (for a review, see Filippenko 1997). On the other hand, Type Ic SNe are devoid of both $\mathrm{H}$ and He features. In the context of spectral properties, the presence of hydrogen in Type Ib/c SNe is still an open question. The absence of hydrogen in early spectra of Type Ib SNe does not imply a complete absence of hydrogen. The absorption feature at $6200 \AA$ in early spectra of these SNe is usually attributed to $\mathrm{H} \alpha$ (Branch et al. 2002; Anupama et al. 2005; Soderberg et al. 2008). Both Branch et al. (2002) and Elmhamdi et al. (2006) have emphasized the existence of hydrogen in Type

* E-mail: mridweeka@aries.res.in, yashasvi04@gmail.com
Ib SNe in different studies conducted on Type Ib SNe. Similar study on Type Ic SNe has also revealed the presence of Helium in some Type Ic SNe (Branch et al. 2006; Elmhamdi et al. 2006). The signatures of $\mathrm{H}$ and $\mathrm{He}$ found in Type $\mathrm{Ib} / \mathrm{c}$ $\mathrm{SNe}$ are due to a thin layer of these elements and their continuous stripping from the progenitor (Valenti et al. 2011).

The progenitors of Type Ib/c SNe lose their hydrogen/helium envelope via strong stellar winds. There are two possible progenitor scenarios proposed for Type Ib/c SNe. One is a massive WR star $\left(>20-25 \mathrm{M}_{\odot}\right)$ which has lost its hydrogen envelope by transfer of mass to a companion star or by stellar winds (Gaskell et al. 1986). Another scenario is a low mass progenitor $\left(>11 \mathrm{M}_{\odot}\right)$ in a binary system (Podsiadlowski et al. 1992; Nomoto et al. 1995; Smartt 2009). A direct identification of progenitor in the pre-SN images is usually a reliable way to distinguish between the different progenitor 
types (Smartt 2009), but previous attempts in this direction for Type Ib/c SNe have been unsuccessful (Crockett et al. 2007; Smartt 2009; Eldridge et al. 2013). However, in one of the most recent study Cao et al. (2013) have reported the possible progenitor identification of iPTF13bvn within a $2 \sigma$ error radius consistent with a massive WR progenitor star. The massive WR progenitor scenario is also supported by the stellar evolutionary models (Groh et al. 2013). Based on observational evidences from early and nebular phase spectroscopy, both a massive WR progenitor as well as an interacting binary progenitor is proposed by several authors (Fremling et al. 2014, 2016; Folatelli et al. 2016; Bersten et al. 2014; Eldridge et al. 2015; Kuncarayakti et al. 2015; Eldridge \& Maund 2016; Hirai 2017a,b). Yoon et al. (2012) have derived the magnitudes of Type Ib/c progenitor stars at pre-SN stage using massive star evolutionary models and found them to be in the range of $\mathrm{M}_{V} \approx-2 \sim$ -3 mag. These are fainter than most of the WR stars in the nearby Universe. Whereas helium star progenitors with low mass in binary systems are brighter in optical domain since they convert into He giant star (Yoon et al. 2012). A similar conclusion is also given by Eldridge et al. (2015) for binary progenitors. In the case of type Ic SNe there is a first possible identification of a progenitor reported for SN 2017ein (Van Dyk et al. 2018).

Some of the Type Ib/c explosions are known to be aspheric. A reasonable degree of polarization and polarization angle, which depends upon the relative size of asymmetry and the sky orientation (Shapiro \& Sutherland 1982; McCall 1984; Hoflich 1991), is reported for these objects. The observed higher degree of polarization in the spectropolarimetric studies during early phase also confirms the asphericity (Wang et al. 2003; Leonard et al. 2006).

In this paper we present the photometry and spectroscopy of MASTER OT J120451.50+265946.6 (hereafter M12045) upto $\sim 250$ days since $B_{\max }$ followed by a detailed analysis and interpretation of the SN characteristics.

\section{DATA ACQUISITION AND REDUCTION}

MASTER Global Robotic Net consists of eight twin robotic telescopes (Lipunov et al. 2010), that work in alert, inspection and survey mode of observations in every night with a clear sky. The main MASTER feature is own real-time autodetection system (Lipunov et al. 2010), that automatically reduces four square degrees in 1-2 minutes after CCD readout. To discover new optical sources (transients) MASTER usually make unfiltered automatic survey in $\mathrm{W}=0.2 \mathrm{~B}+0.8 \mathrm{R}$, calibrated by USNO-B1 R2,B2/R1,B1 thousands field stars. During such survey MASTER-Tunka auto-detection system discovered optical transient (OT) source at RA = $12 \mathrm{~h} 04 \mathrm{~m} 51.50 \mathrm{~s}$ and Dec $=26 \mathrm{~d} 59 \mathrm{~m} 46.6 \mathrm{~s}$ on $2014-10-28.87454$ UT with $13.9 \mathrm{~m}$ unfiltered magnitude (magnitude limit $=$ $18.1 \mathrm{mag})$. This OT was located $4^{\prime \prime}$ West and $13^{\prime \prime}$ North of the center of NGC 4080 galaxy. MASTER has reference image without OT on 2011-04-21.67477 UT with 20.2m unfiltered magnitude limit (Lipunov et al. 2010). The MASTER photometry of the OT is listed in Table A2.

Our multiband observing campaign of M12045 started 17 days after the MASTER discovery and continued upto 210 days since discovery. We used the $104 \mathrm{~cm}$ Sampurnanand
Telescope (ST) (Sagar 1999) and $201 \mathrm{~cm}$ Himalayan Chandra Telescope (HCT) (Prabhu \& Anupama 2010) equipped with the broadband $B V R I$ filters for the photometric monitoring. The images were pre-processed and cleaned using standard IRAF ${ }^{1}$ packages. In all the images psf (point spread function) photometry was done to extract the instrumental magnitudes following the steps described in detail in Singh et al. (2018). Given the location of the SN in the host, the SN flux was contaminated by the host galaxy flux. To estimate the true SN flux free of any host galaxy contamination we performed template subtraction. The templates were observed on February 26, 2017 with $201 \mathrm{~cm}$ HCT under good photometric conditions. One such template subtracted image is shown in figure A1. The instrumental SN magnitude is then measured in the subtracted images.

To calibrate the SN instrumental magnitude, we observed three Landolt equatorial standards (Landolt 2009) PG 0918+029, PG 0942-029 and PG 1525-071 and the SN field on February 26, 2017 under good photometric conditions with seeing $\sim 2$ arcsec in the $V$ band with the $201 \mathrm{~cm}$ HCT. The brightness of the standard stars was in the range $16.4<V<12.27$ and the colour range was $-0.271<B-V$ $<1.109$. The standard fields were observed at different airmasses ranging between 1.6 and 1.2. The standard magnitudes and the instrumental magnitudes of the Landolt stars were used to simultaneously fit for the zero points and the colour coefficients following the least square regression technique (Stetson 1992). Since the Landolt fields were observed only for estimating the zero points and the colour coefficients, we used the site extinction values (Stalin et al. 2008) to correct for the atmospheric extinction. The coefficients thus obtained are used to transform the instrumental magnitudes of the Landolt stars to standard magnitudes. Our calibration shows that the rms scatter between the transformed and standard magnitudes is $\sim 0.06$ in $B$ and $V$ bands and $\sim 0.03$ in $R$ and $I$ bands. Using these transformation equations we generated seven non-variable local standard stars in the SN field (shown in figure A2 and tabulated in Table A1). Using the secondary standards night to night zero-points were determined and SN magnitudes were calibrated differentially. The resultant error in the SN magnitude is obtained by adding in quadrature the photometric and the calibration errors. The calibrated SN magnitudes along with the associated errors are listed in Table A2.

Long slit low resolution spectroscopic data were obtained at 27 epochs from $201 \mathrm{~cm}$ HCT. We used two grisms Gr7 (3800 - $7800 \AA$ A, resolution 1330) and Gr8 (5800 - 9200 $\AA$, resolution 2190) in order to cover the visible region. Arc lamps ( $\mathrm{FeAr}$ and $\mathrm{FeNe}$ ) and spectrophotometric standard stars (Feige 34, Feige 110 and $\mathrm{Hz} 44$ ) were observed each night along with SN for wavelength and flux calibration respectively. Necessary preprocessing and extraction of spectra were done under IRAF environment. The dispersion relations were obtained using the spectra of arc lamp. The dispersion correction was applied to the SN spectra, and OI

1 IRAF stands for Image Reduction and Analysis Facility distributed by the National Optical Astronomy Observatories which is operated by the Association of Universities for research in Astronomy, Inc., under cooperative agreement with the National Science Foundation. 
Table 1. Details of MASTER OT J120451.50+265946.6 and it's host galaxy NGC 4080 .

\begin{tabular}{cc}
\hline \hline Host galaxy & NGC 4080 \\
Galaxy type & SAc C ${ }^{\dagger}$ \\
Constellation & Virgo \\
Redshift & $0.001891 \ddagger$ \\
Major Diameter & $1.50 \mathrm{arcmin}$ \\
Minor Diameter & $0.70 \mathrm{arcmin}$ \\
Helio. Radial Velocity & $567 \pm 4 \mathrm{~km} / \mathrm{sec}$ \\
R.A.(J2000.0) & $12^{h} 04^{m} 51.50^{s}$ \\
Dec.(J2000.0) & $+29^{d} 59^{m} 46.6^{s}$ \\
Distance modulus & $31.43 \mathrm{mag}$ \\
Galactic Extincion E(B-V) & $0.02 \mathrm{mag}$ \\
SN type & Ib \\
Offset from nucleus & $4^{\prime \prime} \mathrm{W}, 13^{\prime \prime} \mathrm{N}$ \\
Date of Discovery & $2456959.375(\mathrm{JD})$ \\
Estimated date of explosion & $29 \mathrm{September,} \mathrm{2014}$ \\
Estimated date of explosion (JD) & 2456929.59 \\
Time of maximum in B band & $2456938.49 \pm 2(\mathrm{JD})$ \\
Time of maximum in V band & $2456948.91 \pm 3(\mathrm{JD})$ \\
\hline$\dagger$ From Ann et al. (2015) ${ }^{\ddagger}$ From Schneider et al. (1990)
\end{tabular}

lines at $5577 \AA$, $6300 \AA$ and $6364 \AA$ were used to cross check the wavelength calibration. In some cases wavelength shift between $0.2 \AA$ to $3 \AA$ was found and corrected. The wavelength corrected spectra were then flux corrected using the observed spectrophotometric standard. The flux calibrated spectra in two grisms Gr7 and Gr8 were combined by applying a scale factor. After wavelength and flux calibration all relative flux spectra were brought to an absolute flux scale using $B V R I$ photometry. Log of spectroscopic observation is given in Table A3.

\section{CHARACTERISTICS OF M12045}

M12045 was discovered by MASTER-Tunka auto-detection system (Lipunov et al. 2010) on 28 October, 2014 22:04 UT in NGC 4080 at an unfiltered magnitude of 13.9 mag (Gress et al. 2014). It was classified as a Type Ib SN, with the presence of well developed HeI, Fe II and Ca II features, a few weeks after maximum (Srivastav et al. 2014b; Terreran et al. 2014). Bright radio emission associated with the SN was reported by Kamble et al. (2014) and Chandra et al. (2014) with the VLA and GMRT, respectively. However no $\mathrm{X}$-ray and UV emission was detected in the Swift observations (Margutti et al. 2014). The basic properties of M12045 and the host galaxy NGC 4080 are listed in Table 1.

To constrain the epoch of maximum light/explosion, we used the spectral identification code SNID (Blondin \& Tonry 2007) and GEneric cLAssification TOol (GELATO) (Harutyunyan et al. 2008) on the first spectrum of M12045 obtained on 29 October, 2014. On the basis of spectral cross correlations with a library of spectral templates, we estimate the explosion epoch to be 29 September, 2014 $(\mathrm{JD}=2456929.59)$. The estimated time of maximum in $B$ and $V$ bands is found to be between 5-9 October, 2014 $(\mathrm{JD}=2456938.49 \pm 2)$ and $15-21$ October, $2014(\mathrm{JD}=$ $2456948.91 \pm 3$ ) respectively. In Table 2 we report the best three matches along with the quality of fit parameter. We have adopted this epoch of $B_{\max }$ for further analysis in the paper.
Table 2. The best three matches to the spectrum obtained on 29 October, 2014 which are adopted for age estimation of M12045.

\begin{tabular}{cccc}
\hline \hline SNID & SN & rlap & $\begin{array}{c}\text { Age since } \mathrm{V}_{\max } \\
\text { (days) }\end{array}$ \\
\hline & SN 1998dt & 12.03 & 7.90 \\
& SN 1990I & 10.58 & 11.40 \\
& SN 1999ex & 9.17 & 9.30 \\
\hline GELATO & SN & Quaity of fit & Estimated phase \\
& & (QoF) & (days) \\
\hline & SN 1999dn & 2.58 & 21.0 (since $B_{\max }$ ) \\
& SN 2008ax & 2.45 & 30.7 (since explosion) \\
& SN 2008ax & 2.31 & 29.0 (since explosion) \\
\hline
\end{tabular}

The host galaxy NGC 4080 of M12045 has three consistent distance estimates available using the Tully-Fisher method (15.0 Mpc $(\mu=30.88)$ Karachentsev et al. 2013, $15.2 \mathrm{Mpc}(\mu=30.91 \pm 0.54)$ Tully et al. 2016 and $16.3 \mathrm{Mpc}$ $(\mu=31.06 \pm 0.43)$ Sorce et al. 2014). We adopt an average distance of $15.5 \mathrm{Mpc}(\mu=30.97 \pm 0.98)$.

The estimation of physical parameters from photometric data depends upon an accurate estimation of extinction both Galactic and host along the line of sight of the SN. The Galactic extinction in the direction of M12045 is found to be $E(B-V)=0.02 \mathrm{mag}$ (Schlafly \& Finkbeiner 2011). Due to the location of Type Ib/c SNe in dusty star forming galaxies, they are expected to suffer from higher host extinction (van Dyk et al. 1996; Anderson \& James 2008; Kelly et al. 2008). From the few initial spectra we find no significant NaID line. However, high resolution spectra are needed to better determine the host galaxy reddening using the NaID line. Drout et al. (2011) has given an alternate method to determine the host extinction using the colors. For a sample of Type Ib/c SNe, Drout et al. (2011) estimate a mean host galaxy reddening of $E(B-V)_{\text {host }}=0.36 \pm 0.24$ for Type Ib/c SNe. Based on our low resolution spectroscopic observations we cannot determine the host reddening correctly. We therefore take the host galaxy reddening value from Drout et al. (2011). We adopt a total (Galactic + host) reddening of $\mathrm{E}(\mathrm{B}-\mathrm{V})=$ $0.38 \pm 0.24$ mag and $\mathrm{R}_{v}=3.1$ (Cardelli et al. 1989) in this work.

In Figure 1 we show the $B V R I$ and $W$ band light curves of M12045. At discovery the SN was a few weeks past maximum. Our observations started $\sim 17$ days after discovery and capture the declining light curves of M12045. We estimate the decline rates between 63 to 245 days since $B_{\max }$ to be $2.29 \pm 0.16,2.45 \pm 0.22$ and $2.50 \pm 0.15 \mathrm{mag} 100 \mathrm{~d}^{-1}$ in $V, R$ and $I$ bands respectively. The decay rate in $B$ band is found to be $0.76 \pm 0.31 \mathrm{mag} 100 \mathrm{~d}^{-1}$ at interval between 63 to 166 days since $B_{\max }$. At late phases the light curves of Type $\mathrm{Ib} \mathrm{SNe}$ are powered by the radioactive decay of ${ }^{56} \mathrm{Co} \rightarrow{ }^{56} \mathrm{Fe}$. The decay rates of M12045 light curves are steeper than the standard ${ }^{56} \mathrm{Co} \rightarrow{ }^{56} \mathrm{Fe}$ decay rates $\left(0.0098 \mathrm{mag}\right.$ day $\left.{ }^{-1}\right) \mathrm{im}-$ plying an optically thin ejecta with inefficient gamma ray trapping. The observed late phase decline rates of M12045 are higher than those found for SNe 1999dn (Benetti et al. 2011) and 2009jf (Sahu et al. 2011) beyond 150 days since $B_{\max }$ (Figure 1; bottom panel).

We construct the quasi bolometric light curve of M12045 by integrating the fluxes between $B$ and $I$ bands. The integrated fluxes at each epoch are converted to luminosity by adopting a distance of $15.5 \mathrm{Mpc}$. Since at late 


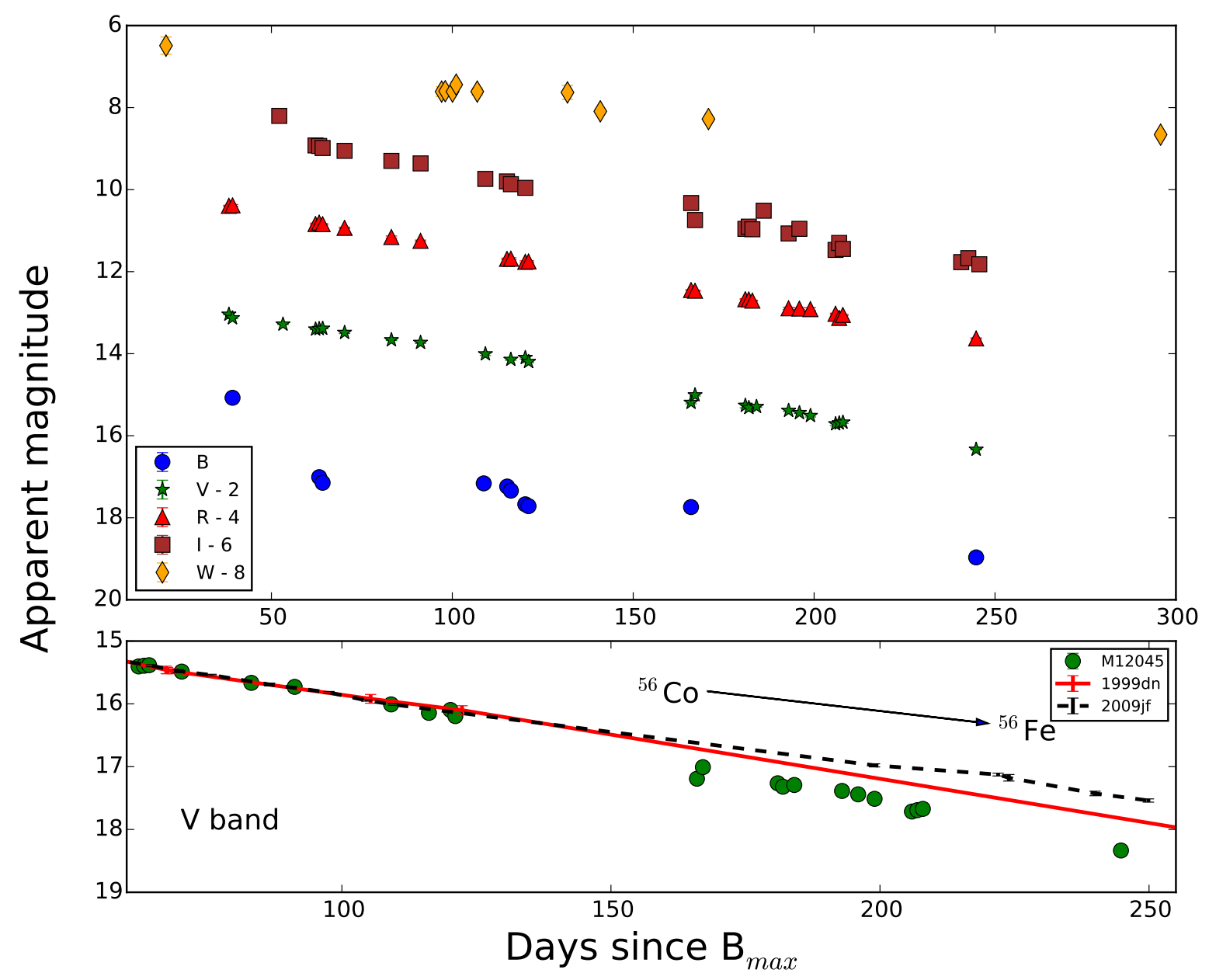

Figure 1. Top panel: $B V R I$ and $W$ band light curve evolution of M12045. Arbitrary offsets are applied in each band for clarity. Bottom panel: The $V$ band light curve of M12045 overplotted with those of SNe 1999dn and 2009jf indicating the faster decline of M12045 beyond 150 days since $B_{\max }$.

phases the contribution from the UV bands is negligible, we only correct for the contribution from the IR bands adopting the numbers given in Prentice et al. (2016). Maeda et al. (2003) and Yamanaka et al. (2015) have given simplified assumptions regarding the fraction of energy deposited due to $\gamma$ rays in the $\mathrm{SN}$ ejecta. Most of the energy in the ejecta is assumed to be generated from ${ }^{56} \mathrm{Co} \rightarrow{ }^{56} \mathrm{Fe}$ radioactive decay. We fit the late phase bolometric light curve of M12045 using the expression given by Yamanaka et al. (2015) described below

$L(t)=M_{56 N i}\left(\left(S_{56 N i}+S_{56 C o}\right) \times\left(1-e^{-\tau}\right)+S_{56 C o} \cdot f_{p}\right)$

where $M_{56 \mathrm{Ni}}$ is the mass of ${ }^{56} \mathrm{Ni}$ ejected during the explosion and $f_{p}$ is the positron fraction. $S_{56 \mathrm{Ni}}$ and $S_{56 \mathrm{Co}}$ are the energy release rates expressed as

$S_{56 N i}=\left(3.90 \times 10^{10}\right) e^{-t / t_{56 N i}} \quad \operatorname{erg} \mathrm{s}^{-1} \mathrm{~g}^{-1}$ and

$S_{56 C o}=\left(7.10 \times 10^{9}\right) \times\left(e^{-t / t_{56 C o}}-e^{-t / t_{56 N i}}\right) \quad \operatorname{erg~s}^{-1} \mathrm{~g}^{-1}$

where $t_{56 \mathrm{Ni}}=8.8$ and $t_{56 \mathrm{Co}}=113.5$ days are the decay time scales. The parameter $\tau$ is defined by

$\tau=1000\left(M_{e j}^{2} / M_{\odot}\right) \times\left(E_{k}^{-1} / 10^{51} \mathrm{erg} \mathrm{s}^{-1}\right)(t / \text { day })^{-2}$

where $M_{e j}$ is mass and $E_{k}$ is the kinetic energy of the ejecta.

We adopt $\tau=5.4$ to fit the late phase bolometric light curve of M12045 and estimate ${ }^{56} \mathrm{Ni}$ to be $0.13 \mathrm{M}_{\odot}{ }^{2}$. A fast declining light curve indicates towards a fainter bolometric

2 If we consider only Galactic reddening the estimated ${ }^{56} \mathrm{Ni}$ is $0.09 \mathrm{M}_{\odot}$. 


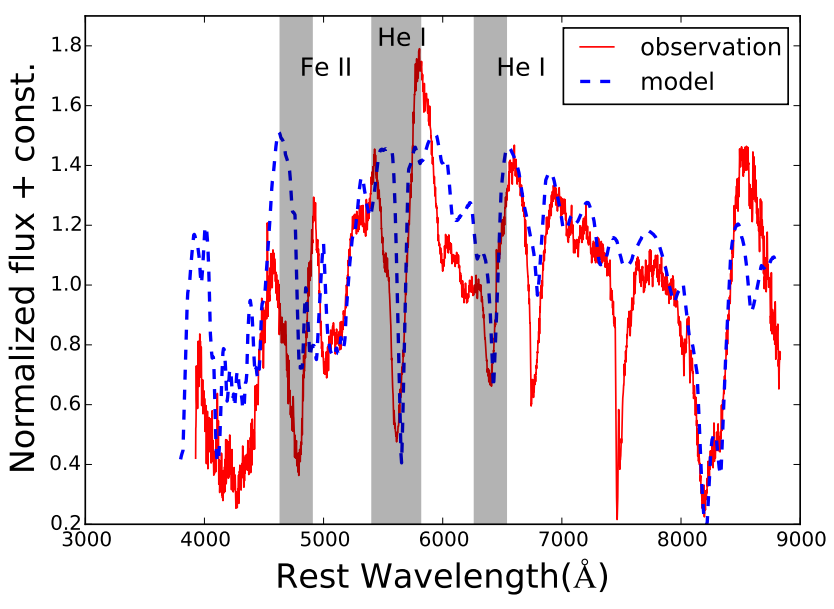

Figure 2. Early spectrum of M12045 along with the SYN++ spectral fitting.

light curve, early commencement of $\gamma$ ray escape and highly mixed ${ }^{56} \mathrm{Ni}$ (Woosley et al. 1994).

\section{KEY SPECTROSCOPIC FEATURES}

In Figure 2 we present the first spectrum of M12045 taken on 29 October, 2014 ( $\sim 20$ days since $B_{\max }$, (Srivastav et al. 2014 b)). The spectrum is characterized by broad P Cygni profile of HeI, Fe II etc. In Figure 2 we also show the best matching synthetic spectrum obtained by $\mathrm{SYN}++$ (Branch et al. 2007; Thomas et al. 2011). The absorption features due to HeI, Fe II multiplet and CaII NIR are well reproduced in the synthetic spectrum. The photospheric velocity associated with the best fit spectrum is $7500 \mathrm{~km} \mathrm{~s}^{-1}$ with a blackbody temperature of $5000 \mathrm{~K}$. The Boltzmann excitation temperature of various lines varies between $10000 \mathrm{~K}$ to 20000K. The spectra fitting is done at four other epochs (20, 34, 40 and 46 days since $B_{\max }$ ) and shown in Figure 3. A gradual decrease in the fit parameters such as velocity and temperature is expected at later phases. The photospheric velocity during this phase varies between $7500 \mathrm{~km} \mathrm{~s}^{-1}$ to $6000 \mathrm{~km} \mathrm{~s}^{-1}$ whereas the blackbody temperature varies between $5000 \mathrm{~K}$ to $4000 \mathrm{~K}$. Due to the blackbody approximation used in $\mathrm{SYN}++$, it is not possible to fit the spectra at very late phases.

\subsection{Spectral Evolution}

The spectral evolution of M12045 during early to late nebular phase ( $\sim 246$ days since discovery) is shown in Figures $4,5,6$. The spectral evolution of M12045 is similar to normal Type Ib SNe. The prominent and noticeable identifying feature of Type Ib SNe is the He I line at $5876 \AA$ which is clearly seen in the spectra of M12045. The other two He features at $6678 \AA$ and $7065 \AA$ are not as prominent as He $5876 \AA$ feature. Fe II feature near $5000 \AA$ is also prominent in the spectral sequence upto $\sim 168$ days since $B_{\max }$. The

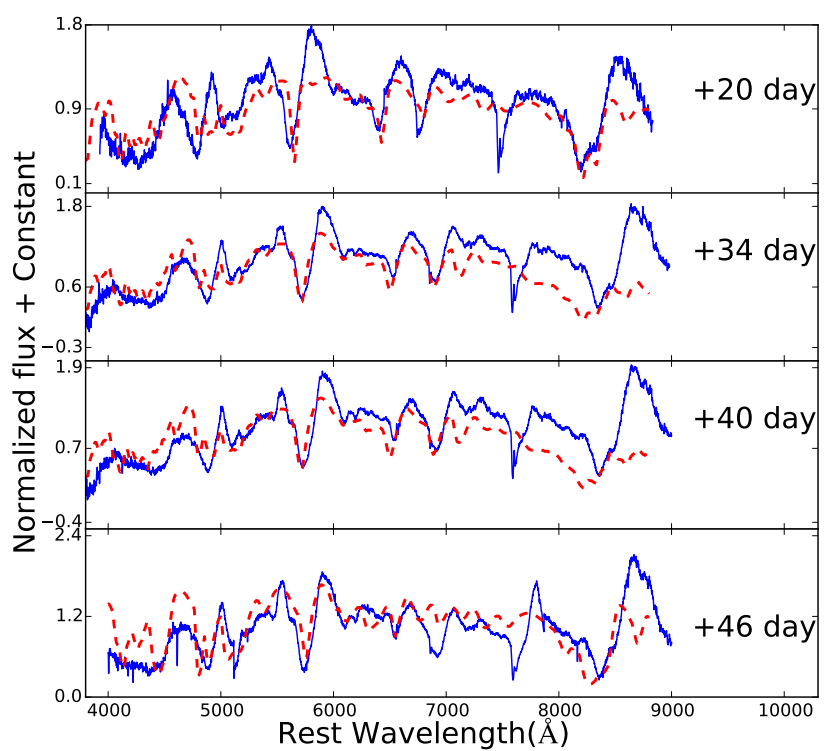

Figure 3. Same as figure 2 and at later epochs.

absorption signature of He I line is stronger than any other absorption feature in the spectral evolution. The absorption trough of CaII NIR triplet is clearly present in the spectral sequence upto $\sim 95$ days since $B_{\max }$ and is very weak thereafter.

To investigate the spectroscopic behaviour of M12045, we have compared the spectral features with other well studied Type Ib SNe. Figure 7 presents early phase (34 days since $\mathrm{B}_{\max }$ ) spectral comparison of M12045 with other Type Ib events such as SNe 1999dn (Benetti et al. 2011), 2005bf (Tominaga et al. 2005; Modjaz et al. 2008, 2014), 2007Y (Stritzinger et al. 2009), 2009jf (Sahu et al. 2011) and iPTF13bvn (Srivastav et al. 2014a).

The spectrum of M12045 is very similar to those of other Type Ib SNe. The P-Cygni profiles of Fe II and HeI lines match very well in all the SNe presented in the Figure 7. The velocity estimated using Fe II and HeI absorption are $9000 \mathrm{~km} \mathrm{~s}^{-1}$ and $13000 \mathrm{~km} \mathrm{~s}^{-1}$, respectively at this epoch. Fe II profile of M12045 matches well with SN 2009jf whereas in the case of SNe 1999dn and 2005bf it is a double peaked structure and in the case of iPTF 13bvn it is a multipeaked profile. He I P-Cygni profile also follows same trend. He I feature near $6678 \AA$ is present in all the SNe in Figure 7 except SN 2009jf.

Figure 5 presents the spectral evolution from 109 to 168 days since $B_{\max }$. As the spectra evolves absorption features of various lines gradually disappear and the emission features become prominent. In the spectral evolution of M12045, [O I] doublet at 6300 and $6363 \AA$ starts appearing after $\sim 79$ days since $B_{\max }$, and is well developed after $\sim 88$ days since $B_{\max }$. The profile of [O I] line is initially flat topped which could be because of the blending of the 6300 and $6363 \AA$ lines. In the later epochs the profile of [O I] lines appears to be asymmetric with bluer component suppressed. Other dominating emission features at nebular phase are MgI] at $4571 \AA$ and [CaII] 7291, $7324 \AA$. There 


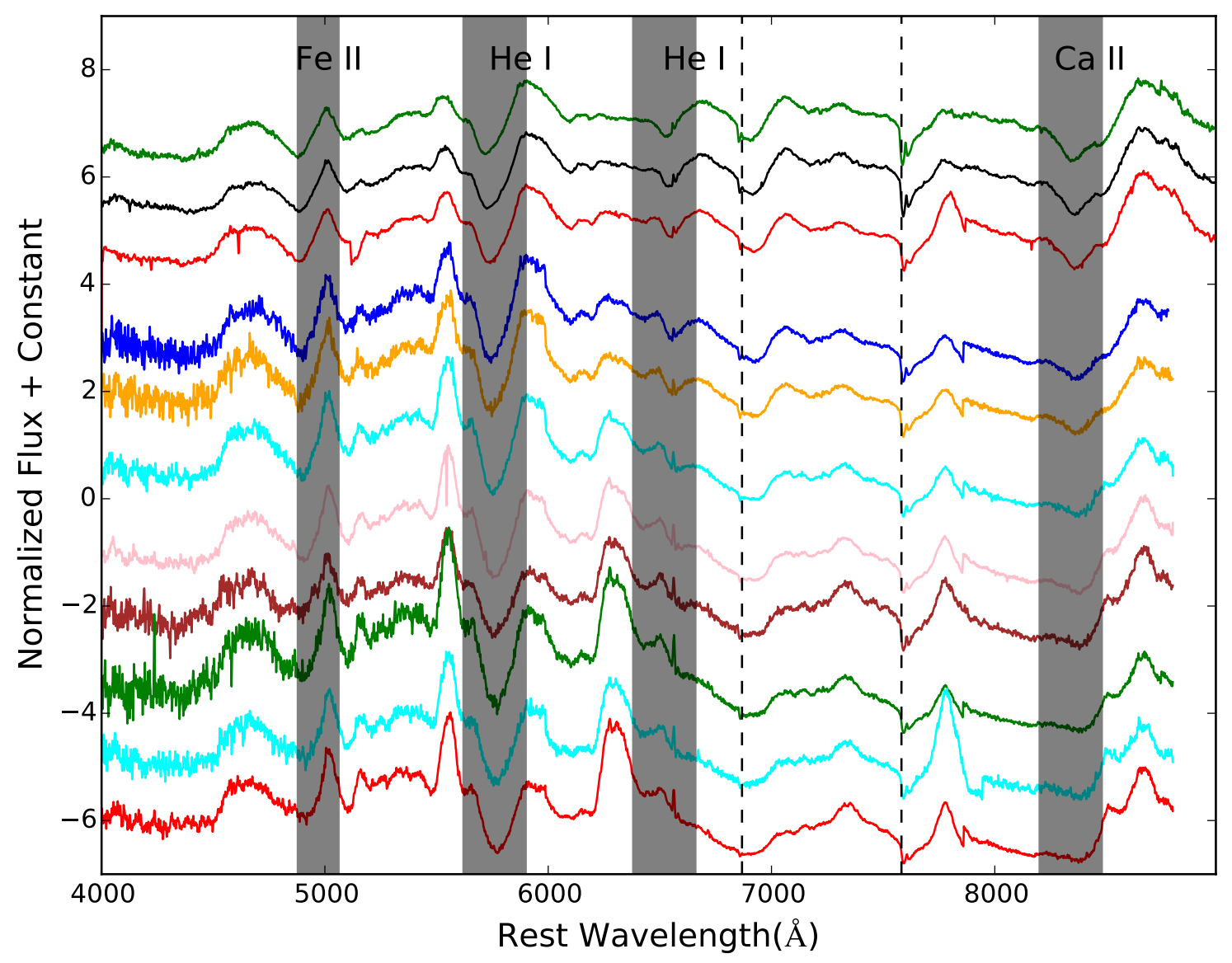

$34.94 d$

$46.94 d$

$54.90 d$

$57.87 d$

$72.84 d$

$79.93 d$

$88.78 d$

$90.80 d$

$95.88 d$

$102.01 d$

Figure 4. Spectral sequence of M12045 during 34 to 102 days since $B_{\max }$. Prime spectral features are shown with shaded regions. Telluric features are also marked in this figure by dashed line.

is possibility of blending of this emission feature with [Fe II] lines at $7155 \AA$, $7172 \AA, 7388 \AA$ and $7452 \AA$. The [Ca II] can also get blended with [O II] emission line at $7320 \AA$ and $7330 \AA$ A.

Figure 6 presents nebular phase spectral sequence during $\sim 177$ to $\sim 246$ days since $B_{\max }$. At these very late epochs ejecta becomes optically thin and the deeper layers of the ejecta can be probed. The spectral sequence during late nebular phase is mostly dominated by forbidden emission features of $\mathrm{Mg}$ I], [O I], [Ca II]. Nebular lines are basically representative of one dimensional line of sight projection for three dimensional distribution of elements, and so contains vital information about the geometry of the ejecta (Maeda et al. 2008; Modjaz et al. 2008; Taubenberger et al. 2009). There are some narrow lines also seen in these spectra, which could be identified as $\mathrm{H} \alpha$, [N II] 6548, $6538 \AA$ and [S II] 6717 , $6731 \AA$ features originated from the underlying H II region (Sahu et al. 2011). Figure 8 presents the spectral comparison of M12045 at 226 days since $B_{\max }$. The profile of [O I] doublet is found to be different in the spectra of objects used for comparison. The profile of [O I] doublet of M12045 shares similarity with SN 2007Y. Whereas SNe 2005bf and 2009jf have clear horned shape/double peaked structure of
[O I] doublet. The double-peaked profile could be due to the explosion geometry. This can also be interpreted as superposition of oxygen lines with high velocity $\mathrm{H} \alpha$ feature (Maurer et al. 2010). The relative strength of [O I] to [CaII] line is found to be different in these spectra. The ratio of $[\mathrm{O} I]$ to [Ca II] line flux is relatively high in case of M12045 and SN 2009jf and low in SN 2005bf and SN 2007Y.

\section{2 $\mathrm{Mg}$ I] line, [O I] doublet}

The late nebular phase spectra of stripped envelope SNe are dominated by the forbidden emission lines for eg. [O I] and [CaII]. The semi-forbidden $\mathrm{MgI}$ ] line (4571 $\AA$ ), which is due to $3 \mathrm{~s}^{2}{ }^{1} \mathrm{~S}_{0}-3 \mathrm{~s} 3 \mathrm{p}^{3} \mathrm{P}_{1}^{0}$ transition, is prominently seen in the spectra of M12045 obtained after $\sim 168$ days since $\mathrm{B}_{\max }$ (Figures 5 and 6). Maeda et al. (2006) suggest that $\mathrm{Mg}$ and $\mathrm{O}$ have similar spatial distribution in the SN ejecta. However, line distributions of $\mathrm{Mg}$ and $\mathrm{O}$ usually deviate from other heavier elements such as Ca or Fe (Mazzali et al. 2005). Figure 9 provides a good opportunity to make a comparison between $\mathrm{MgI}$ ] and [OI] evolution features. In the case of M12045, [O I] profiles are broader than $\mathrm{Mg}$ I] line profiles 


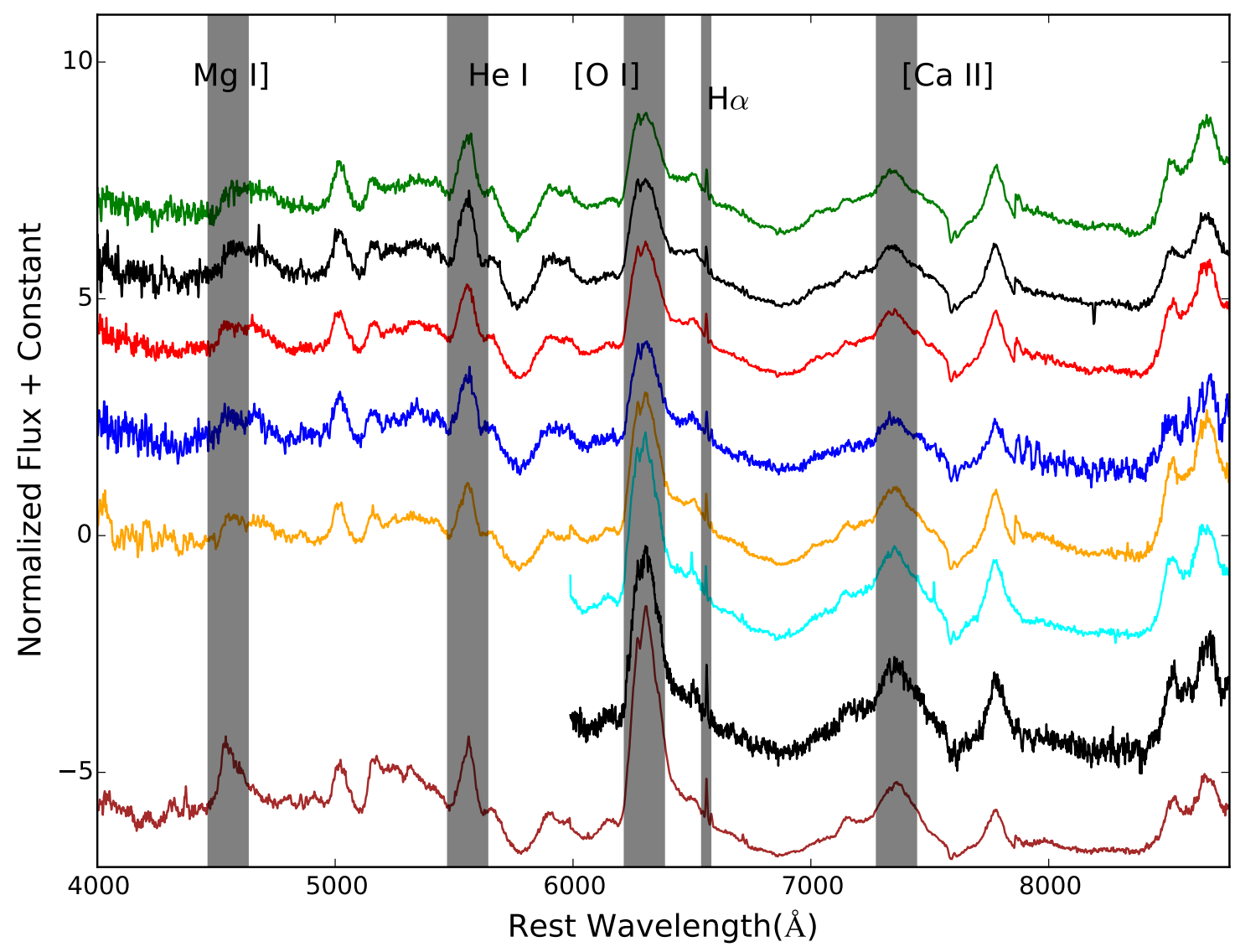

$109.76 d$

$116.01 d$

$120.84 d$

$122.83 d$

$129.82 d$

$137.96 \mathrm{~d}$

$146.75 d$

$168.81 d$

Figure 5. Spectral sequence of M12045 during 109 to 168 days since $B_{\max }$.

which indicates blending with nebular emission lines of other elements. In the present case line profiles are different in terms of the shift from zero velocity. We can see a significant blueshift in the case of $\mathrm{Mg} \mathrm{I}$ ] line profile whereas [O I] doublet profile is slightly redshifted. Blueshift of $\mathrm{Mg}$ I] line is because of residual opacity generated from multiple Fe transitions (Taubenberger et al. 2009). While the slight redshift in the [O I] profile arises mostly due to the asymmetry in the ejecta.

\subsection{Mass of neutral oxygen and $[\mathrm{O} \mathrm{I}] /\left[\begin{array}{ll}\mathrm{Ca} & \mathrm{II}\end{array}\right]$ ratio}

Late nebular phase spectra of Type Ib SNe are enriched with [O I] emission lines. Explosion geometry can be inferred from the [O I] doublet profile. This feature is fairly isolated and unblended. Other prominent late nebular features as [Ca II] and $\mathrm{Mg} \mathrm{I}]$ are contaminated by different nearby line blending. In Figure 9 we present evolution of oxygen profile during late phases. As we discussed earlier in section 4.1, [O I] doublet profile is asymmetric with the suppression of blue component. In Figure 9 we see a shifted redward component from the zero velocity. These signatures suggest that the explosion of M12045 is axisymmetric. This geometry is linked with oxygen distributed as a torus viewed from the equatorial direction or a blob of oxygen moving perpendicular to the line of sight (Mazzali et al. 2001; Maeda et al. 2002, 2006).

By measuring the flux of the $[\mathrm{O} \mathrm{I}]$ line, we can estimate the mass of neutral oxygen following the expression given by Uomoto (1986) as described below.

$M_{O}=10^{8} \times D^{2} \times F([\mathrm{O} \mathrm{I}]) \times e^{\left(2.28 / T_{4}\right)}$

where $\mathrm{M}_{O}$ is mass of neutral oxygen in term of $\mathrm{M}_{\odot}, \mathrm{D}$ is the distance to the $\mathrm{SN}$ in $\mathrm{Mpc}, \mathrm{F}([\mathrm{OI}])$ is the observed absolute flux of [OI] line in erg s $\mathrm{s}^{-1} \mathrm{~cm}^{-2}$ and $\mathrm{T}_{4}$ is the temperature associated with oxygen emitting region in units of $10^{4}$ $\mathrm{K}$. The above equation holds good in high density regime $\left(\mathrm{N}_{e} \geq 10^{6} \mathrm{~cm}^{-3}\right)$, associated with the ejecta of SNe Type Ib (Schlegel \& Kirshner 1989; Gomez \& Lopez 1994; Elmhamdi et al. 2004). Temperature of the line emitting region can be estimated using flux ratio of [O I] 5577/6300-6344 lines. In the observed spectra of M12045 during the late nebular phase, [O I] $5577 \AA$ line is not clearly detected and hence an upper limit of $\sim 0.1$ can be set on the ratio of $[\mathrm{OI}]$ 5577/6300-6364 line flux. For this limit, the line emitting region can be either a low temperature $\left(\mathrm{T}_{4} \leq 0.4\right)$, high density region or a high temperature region $\left(\mathrm{T}_{4}=1\right)$ with low electron density $\left(\mathrm{n}_{e} \leq 5 \times 10^{6} \mathrm{~cm}^{-3}\right.$ Maeda et al. 2007). 


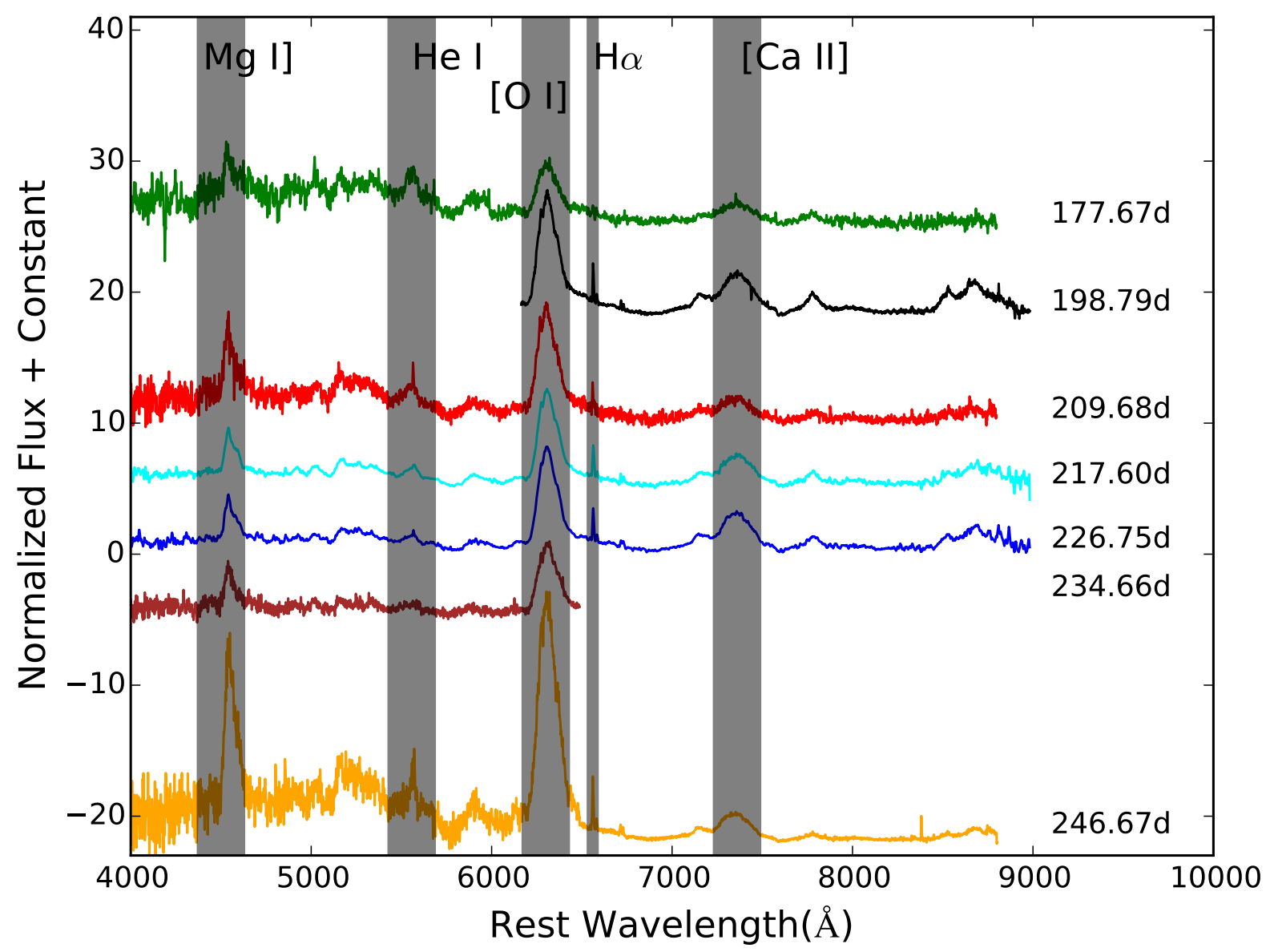

Figure 6. Nebular phase spectra of M12045 during 177 to 246 days since $B_{\max }$. This spectral sequence is dominated by emission features of various lines indicated by shaded regions.

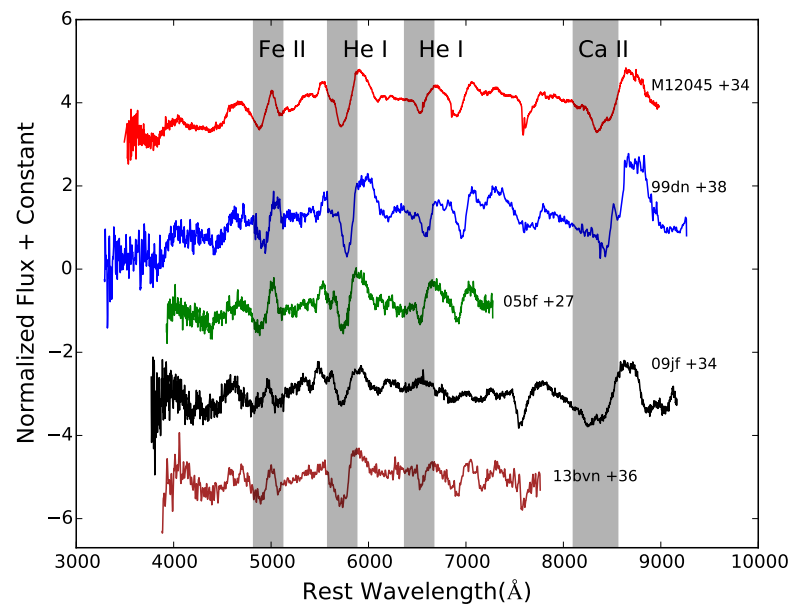

Figure 7. Comparison of spectral features of M12045 at 34 days since $\mathrm{B}_{\max }$ with other well studied Type Ib SNe. Prominent He feature is present in all the SNe shown here.
In the $\sim 226 \mathrm{~d}$ spectrum, the observed flux of [O I] line, $\mathrm{F}([\mathrm{O} \mathrm{I}])=1.25 \times 10^{-13} \mathrm{erg} \mathrm{s}^{-1} \mathrm{~cm}^{-2}$, distance of $15.5 \mathrm{Mpc}$, and considering the case of low temperature $\left(\mathrm{T}_{4}=0.4\right)$, the estimated mass of neutral oxygen is $0.90 \mathrm{M}_{\odot}$.

The prime cause of [O I] emission is the formation of a layer of oxygen during the hydrostatic burning phase. The main sequence mass of the progenitor is directly related with the ejected mass of oxygen. Thielemann et al. (1996) have predicted nucleosynthesis yields for progenitor masses between 13-25 $\mathrm{M}_{\odot}$ based on nucleosynthesis calculations. Based on the calculations, Thielemann et al. (1996) showed that for oxygen masses $0.22,0.43,1.48$, and $3.00 \mathrm{M}_{\odot}$, the associated progenitor mass should be $13,15,20$ and $25 \mathrm{M}_{\odot}$ respectively. For 13,15 and $25 \mathrm{M}_{\odot}$ progenitor masses, the corresponding He core mass estimates given by Thielemann et al. (1996) were 3.3, 4.0 and $8.0 \mathrm{M}_{\odot}$ respectively. The estimated oxygen mass of $0.90 \mathrm{M}_{\odot}$ in M12045 indicates towards a main sequence progenitor mass of $15-20 \mathrm{M}_{\odot}$ with a He core mass between $4-8 \mathrm{M}_{\odot}$.

Using the ratio of [O I] and [Ca II] line fluxes we can estimate the main sequence mass of the progenitor. The ratio of the fluxes of $\left[\mathrm{OI}_{\mathrm{I}}\right]$ and $[\mathrm{Ca} \mathrm{II}]$ lines is found to be insensitive 


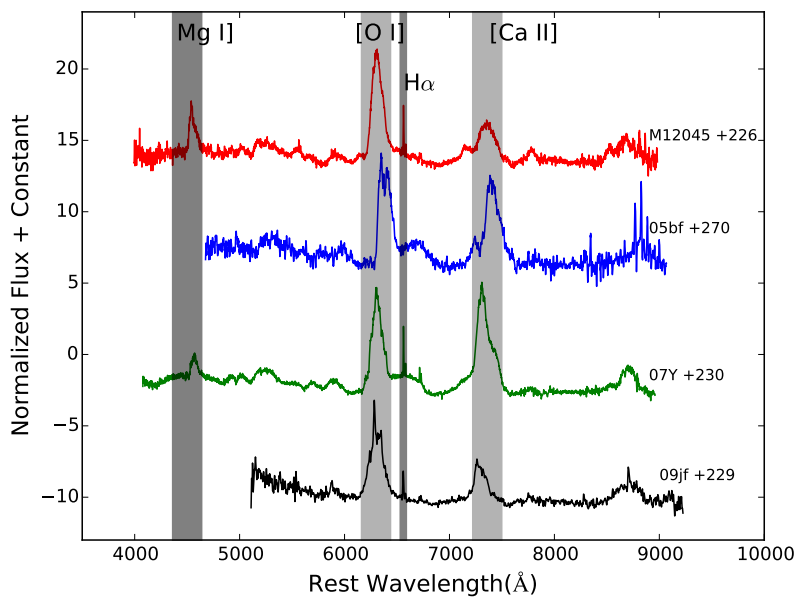

Figure 8. Comparison of spectral features of M12045 at 226 days since $\mathrm{B}_{\max }$ with other well studied Type Ib SNe. Due to limited wavelength coverage blue spectral regime is not available for $\mathrm{SNe}$ 2005bf and 2009jf. The [O I] line profile in M12045 shares a similarity with SN 2007Y. [Ca II] doublet is clearly present in M12045 as well as in the comparison SNe.

to density, temperature whereas it increases with an increasing progenitor mass (Fransson \& Chevalier 1989; Elmhamdi et al. 2004). In the case of M12045 this line ratio is between 2.1 to 2.5 for spectra between $\sim 168$ to 226 days since $B_{\text {max }}$. This line ratio is nearly constant for SNe post $\sim 280$ days since explosion (Elmhamdi et al. 2004). Kuncarayakti et al. (2015) have compared this ratio for a number of stripped envelope $\mathrm{SNe}$ and found that the line ratio for Type II SNe does not exceed 0.7 while there is a considerable spread seen for Type Ib/c SNe ( 0.9 to 2.5$)$. As stated in Kuncarayakti et al. (2015), this spread in line ratio indicates towards two different progenitor populations for Type Ib/c SNe - those coming from massive WR stars and those from lower mass progenitors in binary systems. The flux ratio of M12045 indicates that it is associated with a massive star progenitor. The line ratios in SNe 1999dn and 2009jf were found to be $\sim 2$ and $\sim 1.82$, respectively with an associated progenitor mass of $\sim 25 \mathrm{M}_{\odot}$ (Benetti et al. 2011; Sahu et al. 2011). The estimated mass of neutral oxygen in M12045 also indicates the progenitor mass between to be $15-20 \mathrm{M}_{\odot}$. Based on the above two estimates we can say that a massive star of main sequence mass $\sim 20 \mathrm{M}_{\odot}$ is likely the progenitor of M12045.

\subsection{Velocity evolution}

We estimated velocities of a few lines from the absorption minima of P-cygni features. Figure 10 presents the velocity evolution of Fe II $\lambda 5169 \AA$, He I $\lambda 5876 \AA$ and Ca II NIR triplet in M12045. A comparison with a few well studied Type Ib SNe is also shown (SNe 2007Y Stritzinger et al. 2009, 2009jf Sahu et al. 2011 and iPTF13bvn Srivastav et al. 2014a) in Figure 10. During the first few days the line velocities for M12045 declines rapidly and then attains a relatively constant value. The velocities estimated using Fe II $5169 \AA$, He I 5876 and Ca II NIR triplet at $\sim 20$ days since $B_{\max }$ are $\sim 8700,13000$ and $8300 \mathrm{~km} \mathrm{~s}^{-1}$, respectively. The velocity
Table 3. Flux measurements of various emission lines in the nebular spectrum $\left(\sim 226\right.$ days since $\left.B_{\max }\right)$ of M12045.

\begin{tabular}{ccc}
\hline \hline Species & $\begin{array}{c}\text { Wavelength } \\
(\AA)\end{array}$ & $\begin{array}{c}\text { Flux } \\
\left(10^{-17}\right)\left(\operatorname{erg~s}^{-1} \mathrm{~cm}^{-2}\right)\end{array}$ \\
\hline H $\alpha$ & 6563 & 254 \\
NII & 6583 & 65 \\
SII & 6717 & 31 \\
SII & 6731 & 22 \\
\hline
\end{tabular}

of Fe II line at $5169 \AA$ at $\sim 20$ days since $B_{\max }$ is lower than what we expect at maximum and is consistent with the average photospheric velocities between $8000 \pm 2000 \mathrm{~km}$ $\mathrm{s}^{-1}$ for Type Ib/c SNe at maximum (Cano 2013). At $\sim 35$ days since $B_{\max }$ these velocities drop to $\sim 4700,7500$ and $5500 \mathrm{~km} \mathrm{~s}^{-1}$. Beyond $\sim 46$ days since $B_{\max }$ the line velocities of Fe II and He I remain nearly constant at $\sim 5000 \mathrm{~km}$ $\mathrm{s}^{-1}$, whereas Ca II line velocity further declines linearly to $\sim 3000 \mathrm{~km} \mathrm{~s}^{-1}$ at 137 days since $B_{\max }$.

Figure 10 (b) shows the comparison of Fe II line at $5169 \AA$ with SNe 2007Y, 2009jf and iPTF 13bvn. At $\sim 20$ days since $B_{\max }$ the velocity of M12045 is higher than all the comparison $\mathrm{SNe}$. At later phases velocity associated with Fe II line at $5169 \AA$ for M12045 is $\sim 1500 \mathrm{~km} \mathrm{~s}^{-1}$ lower than that of SN 2009jf and $1700 \mathrm{~km} \mathrm{~s}^{-1}$ higher than velocities associated with SN 2007Y. On the basis of the available data we can see that the velocity of the CaII NIR for M12045 is lower than SN 2009jf and higher than SNe 2007Y and iPTF13bvn (Figure 10 (c)). At $\sim 20$ days since $B_{\max }$ the velocity of the HeI line for M12045 is higher than the rest of the comparison sample (Figure 10 (d)). Whereas at later phases the velocity of the He I line for M12045 seems to be lower than those of other SNe.

\section{HOST GALAXY METALLICITY}

In the nebular spectrum of M12045, narrow emission lines are seen (Figure 6). The observed narrow lines originate from the $\mathrm{H}$ II region in which the $\mathrm{SN}$ is embedded. Using the flux of the narrow emission lines, we can estimate the metallicity at the SN location in the host galaxy. There are several diagnostics for metallicity measurements given by several authors (McGaugh 1991; Kewley \& Dopita 2002; Pettini \& Pagel 2004; Pilyugin \& Thuan 2005) which depend upon the various emission lines ratios and calibrations. However, due to limitation of the observed wavelength range $(\sim 4000$ - $9000 \AA$ ) and non-detection/very weak [OIII] lines, we are left with only N2 index calibration of Pettini \& Pagel (2004) for estimating the metallicity of the SN region. In Table 3 we list the flux measurements of nebular emission lines seen in the $\sim 226 \mathrm{~d}$ spectrum of M12045. Based on the N2 index we estimate the metallicity to be $12+\log (\mathrm{O} / \mathrm{H})=8.56 \pm 0.18$ dex.

The value of oxygen abundance $12+\log (\mathrm{O} / \mathrm{H})$ for the Sun available in the literature are $8.69 \pm 0.05$ dex (Allende Prieto et al. 2001), 8.69 dex (Asplund et al. 2009) and $8.76 \pm 0.07$ dex (Caffau et al. 2011). The oxygen abundance estimated for the SN region is less than the Solar value, indicating marginally sub-Solar metallicity at the SN region within the host galaxy NGC 4080. The sub-Solar metallicity environment of M12045 is consistent with a massive WR 


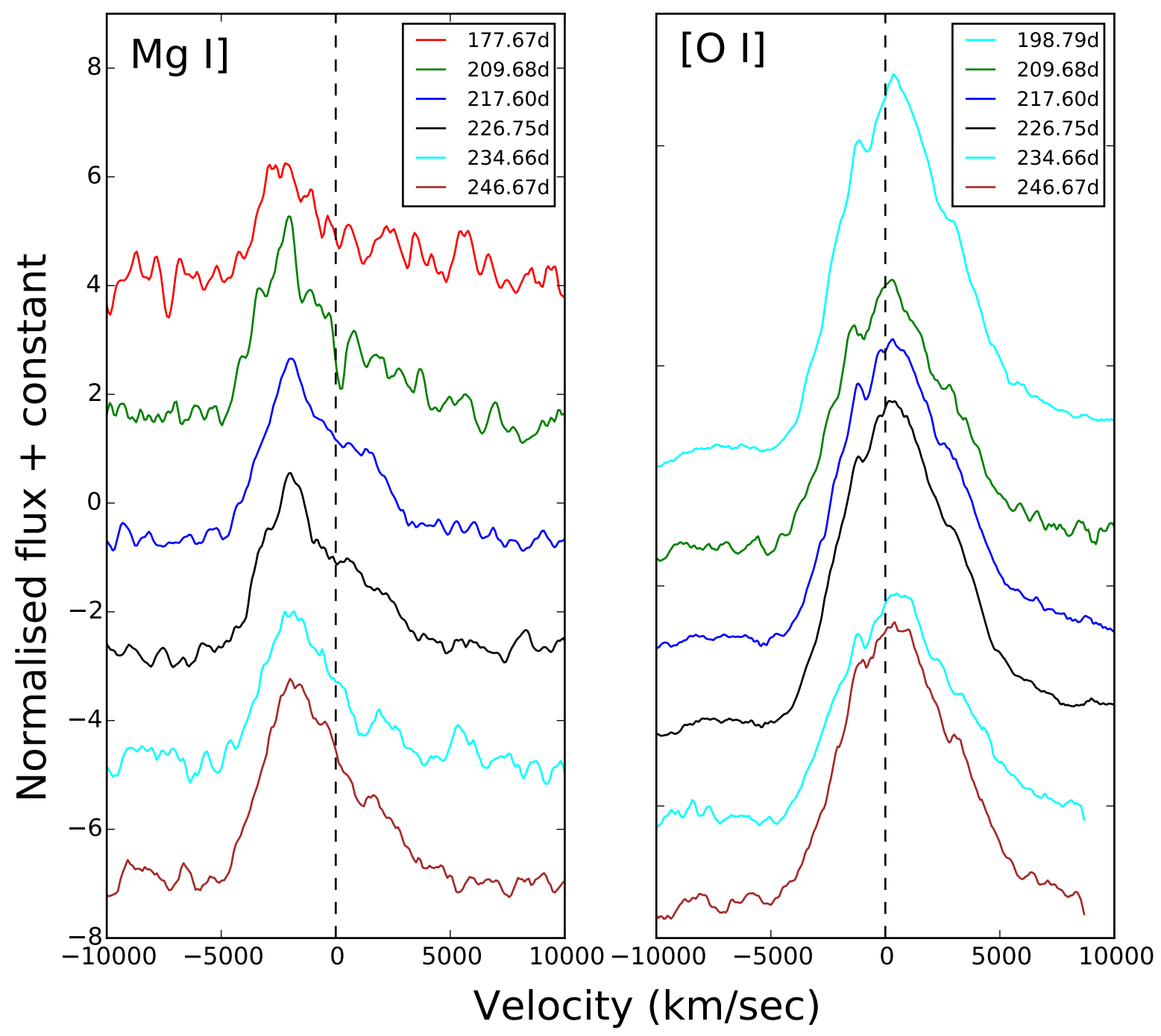

Figure 9. Mg I] and [O I] line evolution in nebular phase of M12045. A prominent difference can be seen in both the profiles in terms of the shift from zero velocity.

progenitor star scenario. The probability which favours a massive WR star as a progenitor system increases with increasing metallicity because stellar winds are metallicity dependent (López-Sánchez \& Esteban 2010).

\section{SUMMARY}

In the present work we discuss the results obtained for a Type Ib SN M12045 based on the photometric and spectroscopic observations. M12045 is one of the few Type Ib SNe with a rich spectroscopic data set spanning upto $\sim 250$ days since $B_{\max }$. Our analysis shows that the SN was discovered a few weeks after maximum and we have been therefore able to capture the linearly declining light curves in $B V R I$ bands. The late phase decline rate in all the bands is considerably steeper than the expected ${ }^{56} \mathrm{Co}$ to ${ }^{56} \mathrm{Fe}$ decay indicating an optically thin ejecta with inefficient gamma ray trapping. Fitting a late phase energy deposition function to the bolometric light curve of M12045 yields a ${ }^{56} \mathrm{Ni}$ mass of $0.13 \mathrm{M}_{\odot}$. Faster decline in the light curve during radioactive tail indicates towards a higher mixing of ${ }^{56} \mathrm{Ni}$ and hence a smaller ${ }^{56} \mathrm{Ni}$ production. The spectroscopic evolution of M12045 is similar to a typical Type Ib SN with enriched features of $\mathrm{He}, \mathrm{O}, \mathrm{Mg}$ and $\mathrm{Ca} I \mathrm{NIR}$. The [O I] doublet profile in the late nebular phase shows asymmetry in the explosion. The spectral sequence of M12045 also shows narrow $\mathrm{H} \alpha$ feature originating from the underlying H II region. Photospheric velocity measured from Fe II line at $5169 \AA$ is consistent with the average velocity of Type Ib/c SNe. The mass of neutral oxygen, the flux ratio of $[\mathrm{OI}]$ and [CaII] lines and sub-Solar metallicity suggests that the progenitor of M12045 

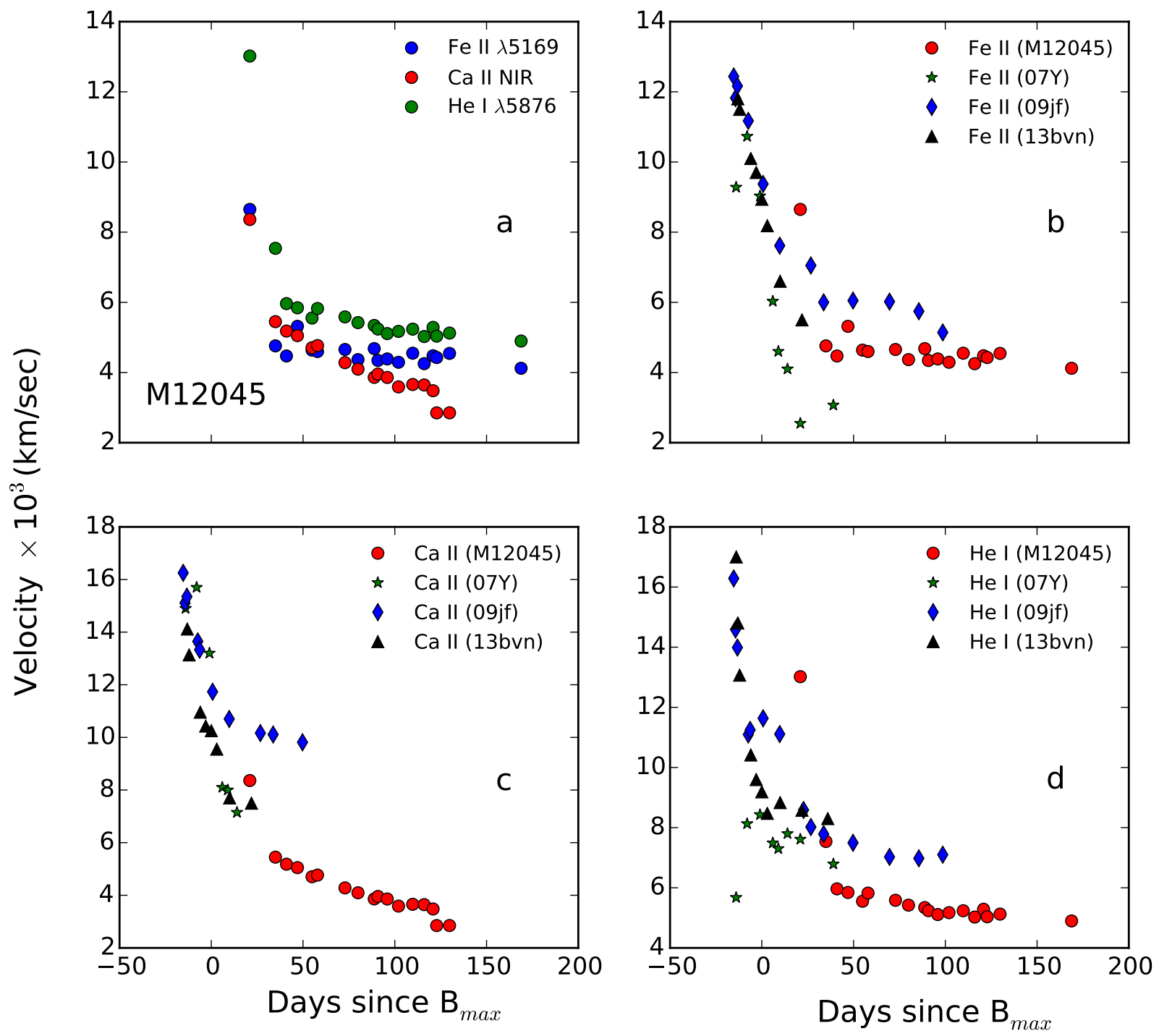

Figure 10. The temporal evolution of the velocities of Fe II 5169 subplot. For the same lines we show a comparison of velocities of M12045 with SNe 2007Y (Stritzinger et al. 2009), 2009jf (Sahu et al. 2011) and iPTF 13bvn (Srivastav et al. 2014a) in the remaining three panels.

could be a massive WR star with a zero age main sequence mass of $\sim 20 \mathrm{M}_{\odot}$.

\section{ACKNOWLEDGMENTS}

We thank the observing staff and observing assistants at $104 \mathrm{~cm} \mathrm{ST}$ and $201 \mathrm{~cm} \mathrm{HCT} \mathrm{for} \mathrm{their} \mathrm{support} \mathrm{during} \mathrm{obser-}$ vations of M12045. We acknowledge Wiezmann Interactive Supernova data REPository http://wiserep.weizmann.ac.il (WISeREP) (Yaron \& Gal-Yam 2012). This research has made use of the CfA Supernova Archive, which is funded in part by the National Science Foundation through grant AST 0907903. This research has made use of the NASA/IPAC Extragalactic Database (NED) which is operated by the Jet Propulsion Laboratory, California Institute of Technology, under contract with the National Aeronautics and Space Administration. KM acknowledges the support from IUSSTF WISTEMM fellowship and UC Davis. SBP and KM acknowledges BRICS grant DST/IMRCD/BRICS/Pilotcall/ProFCheap $/ 2017(\mathrm{G})$ for the present work. BK acknowledges the Science and Engineering Research Board (SERB) under the Department of Science \& Technology, Govt. of India, for financial assistance in the form of National Post-Doctoral Fellowship (Ref. no. PDF/2016/001563). DKS, GCA and BK acknowledge BRICS grant DST/IMRCD/BRICS/PilotCall1/MuMeSTU/2017(G) 
for the present work. MASTER equipment acknowledges to Lomonosov Moscow State University Development Program. VL acknowledge BRICS grant 17-52-80133. PB work was supported by RNF16-12-00085. SB acknowledges NSFC Project 11573003 and also is partially supported by China postdoctoral science foundation grant No. 2018T110006.

\section{REFERENCES}

Allende Prieto C., Lambert D. L., Asplund M., 2001, ApJ, 556, L63

Anderson J. P., James P. A., 2008, MNRAS, 390, 1527

Ann H. B., Seo M., Ha D. K., 2015, ApJS, 217, 27

Anupama G. C., Sahu D. K., Deng J., Nomoto K., Tominaga N., Tanaka M., Mazzali P. A., Prabhu T. P., 2005, ApJ, 631, L125

Asplund M., Grevesse N., Sauval A. J., Scott P., 2009, ARA\&A, 47, 481

Benetti S., et al., 2011, MNRAS, 411, 2726

Bersten M. C., et al., 2014, AJ, 148, 68

Blondin S., Tonry J. L., 2007, ApJ, 666, 1024

Branch D., et al., 2002, ApJ, 566, 1005

Branch D., Jeffery D. J., Young T. R., Baron E., 2006, PASP, 118, 791

Branch D., Parrent J., Troxel M. A., Casebeer D., Jeffery D. J., Baron E., Ketchum W., Hall N., 2007, in di Salvo T., Israel G. L., Piersant L., Burderi L., Matt G., Tornambe A., Menna M. T., eds, American Institute of Physics Conference Series Vol. 924, The Multicolored Landscape of Compact Objects and Their Explosive Origins. pp 342-349, doi:10.1063/1.2774879

Caffau E., Ludwig H.-G., Steffen M., Freytag B., Bonifacio P., 2011, Sol. Phys., 268, 255

Cano Z., 2013, MNRAS, 434, 1098

Cao Y., et al., 2013, ApJ, 775, L7

Cardelli J. A., Clayton G. C., Mathis J. S., 1989, ApJ, 345, 245

Chandra P., Nayana A. J., Ray A., Yadav N., Chakraborti S., 2014, The Astronomer's Telegram, 6755

Crockett R. M., et al., 2007, MNRAS, 381, 835

Drout M. R., et al., 2011, ApJ, 741, 97

Eldridge J. J., Maund J. R., 2016, MNRAS, 461, L117

Eldridge J. J., Fraser M., Smartt S. J., Maund J. R., Crockett R. M., 2013, MNRAS, 436, 774

Eldridge J. J., Fraser M., Maund J. R., Smartt S. J., 2015, MNRAS, 446, 2689

Elmhamdi A., Danziger I. J., Cappellaro E., Della Valle M., Gouiffes C., Phillips M. M., Turatto M., 2004, A\&A, 426, 963

Elmhamdi A., Danziger I. J., Branch D., Leibundgut B., Baron E., Kirshner R. P., 2006, A\&A, 450, 305

Filippenko A. V., 1997, ARA\&A, 35, 309

Folatelli G., et al., 2016, ApJ, 825, L22

Fransson C., Chevalier R. A., 1989, ApJ, 343, 323

Fremling C., et al., 2014, A\&A, 565, A114

Fremling C., et al., 2016, A\&A, 593, A68

Gaskell C. M., Cappellaro E., Dinerstein H. L., Garnett D. R., Harkness R. P., Wheeler J. C., 1986, ApJ, 306, L77

Gomez G., Lopez R., 1994, AJ, 108, 195

Gress O., et al., 2014, The Astronomer's Telegram, 6634

Groh J. H., Georgy C., Ekström S., 2013, A\&A, 558, L1

Harutyunyan A. H., et al., 2008, A\&A, 488, 383

Hirai R., 2017a, MNRAS, 466, 3775

Hirai R., 2017b, MNRAS, 469, L94

Hoflich P., 1991, A\&A, 246, 481

Kamble A., Soderberg A., Margutti R., Parrent J., Milisavljevic D., 2014, The Astronomer's Telegram, 6712
Karachentsev I. D., Makarov D. I., Kaisina E. I., 2013, AJ, 145, 101

Kelly P. L., Kirshner R. P., Pahre M., 2008, ApJ, 687, 1201

Kewley L. J., Dopita M. A., 2002, ApJS, 142, 35

Kuncarayakti H., et al., 2015, A\&A, 579, A95

Landolt A. U., 2009, AJ, 137, 4186

Leonard D. C., et al., 2006, Nature, 440, 505

Lipunov V., et al., 2010, Advances in Astronomy, 2010, 349171

López-Sánchez Á. R., Esteban C., 2010, A\&A, 516, A104

Maeda K., Nakamura T., Nomoto K., Mazzali P. A., Patat F., Hachisu I., 2002, ApJ, 565, 405

Maeda K., Mazzali P. A., Deng J., Nomoto K., Yoshii Y., Tomita H., Kobayashi Y., 2003, ApJ, 593, 931

Maeda K., Nomoto K., Mazzali P. A., Deng J., 2006, ApJ, 640, 854

Maeda K., et al., 2007, ApJ, 666, 1069

Maeda K., et al., 2008, Science, 319, 1220

Margutti R., Brown P. J., Kamble A., Milisavljevic D., Parrent J., Soderberg A. M., 2014, The Astronomer's Telegram, 6719

Maurer I., Mazzali P. A., Taubenberger S., Hachinger S., 2010, MNRAS, 409, 1441

Mazzali P. A., Nomoto K., Patat F., Maeda K., 2001, ApJ, 559, 1047

Mazzali P. A., et al., 2005, Science, 308, 1284

McCall M. L., 1984, MNRAS, 210, 829

McGaugh S. S., 1991, ApJ, 380, 140

Modjaz M., Kirshner R. P., Blondin S., Challis P., Matheson T., 2008, ApJ, 687, L9

Modjaz M., et al., 2014, AJ, 147, 99

Nomoto K. I., Iwamoto K., Suzuki T., 1995, Phys. Rep., 256, 173

Pettini M., Pagel B. E. J., 2004, MNRAS, 348, L59

Pilyugin L. S., Thuan T. X., 2005, ApJ, 631, 231

Podsiadlowski P., Joss P. C., Hsu J. J. L., 1992, ApJ, 391, 246

Prabhu T. P., Anupama G. C., 2010, in Astronomical Society of India Conference Series.

Prentice S. J., et al., 2016, MNRAS, 458, 2973

Sagar R., 1999, Current Science, 77, 643

Sahu D. K., Gurugubelli U. K., Anupama G. C., Nomoto K., 2011, MNRAS, 413, 2583

Schlafly E. F., Finkbeiner D. P., 2011, ApJ, 737, 103

Schlegel E. M., Kirshner R. P., 1989, AJ, 98, 577

Schneider S. E., Thuan T. X., Magri C., Wadiak J. E., 1990, ApJS, 72, 245

Shapiro P. R., Sutherland P. G., 1982, ApJ, 263, 902

Singh M., et al., 2018, MNRAS, 474, 2551

Smartt S. J., 2009, ARA\&A, 47, 63

Soderberg A. M., et al., 2008, Nature, 453, 469

Sorce J. G., Tully R. B., Courtois H. M., Jarrett T. H., Neill J. D., Shaya E. J., 2014, MNRAS, 444, 527

Srivastav S., Anupama G. C., Sahu D. K., 2014a, MNRAS, 445, 1932

Srivastav S., Sahu D. K., Anupama G. C., 2014b, The Astronomer's Telegram, 6639

Stalin C. S., Hegde M., Sahu D. K., Parihar P. S., Anupama G. C., Bhatt B. C., Prabhu T. P., 2008, Bulletin of the Astronomical Society of India, 36, 111

Stetson P. B., 1992, J. R. Astron. Soc. Canada, 86, 71

Stritzinger M., et al., 2009, ApJ, 696, 713

Taubenberger S., et al., 2009, MNRAS, 397, 677

Terreran G., et al., 2014, The Astronomer's Telegram, 6641

Thielemann F.-K., Nomoto K., Hashimoto M.-A., 1996, ApJ, 460, 408

Thomas R. C., Nugent P. E., Meza J. C., 2011, PASP, 123, 237

Tominaga N., et al., 2005, ApJ, 633, L97

Tully R. B., Courtois H. M., Sorce J. G., 2016, AJ, 152, 50

Uomoto A., 1986, ApJ, 310, L35

Valenti S., et al., 2011, MNRAS, 416, 3138

Van Dyk S. D., et al., 2018, ApJ, 860, 90 
Wang L., Baade D., Höflich P., Wheeler J. C., 2003, ApJ, 592, 457

Woosley S. E., Eastman R. G., Weaver T. A., Pinto P. A., 1994, ApJ, 429, 300

Yamanaka M., et al., 2015, ApJ, 806, 191

Yaron O., Gal-Yam A., 2012, PASP, 124, 668

Yoon S.-C., Gräfener G., Vink J. S., Kozyreva A., Izzard R. G., 2012, A\&A, 544, L11

van Dyk S. D., Hamuy M., Filippenko A. V., 1996, AJ, 111, 2017

\section{APPENDIX A: LOG OF PHOTOMETRIC AND} SPECTROSCOPIC OBSERVATIONS

Figure A1 shows one of the template subtracted SN image. The local standards used for calibration along with the SN location are marked in Figure A2 and listed in Table A1. The calibrated SN magnitudes and the corresponding errors in BVRI filters are listed in Table A2. A complete log of spectroscopic observations is given in Table A3. 

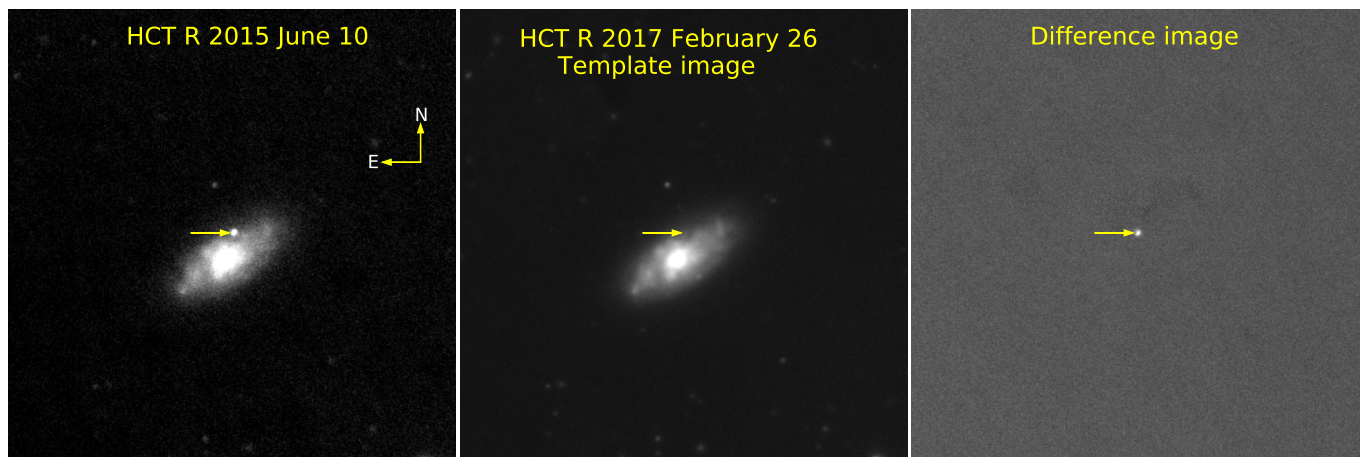

Figure A1. This figure consists of three panels. Left panel is the image acquired with $201 \mathrm{~cm}$ HCT in R band along with the location of SN marked. Middle panel represents the template image which is obtained on February 26, 2017. Right panel shows the difference image received after template subtraction.

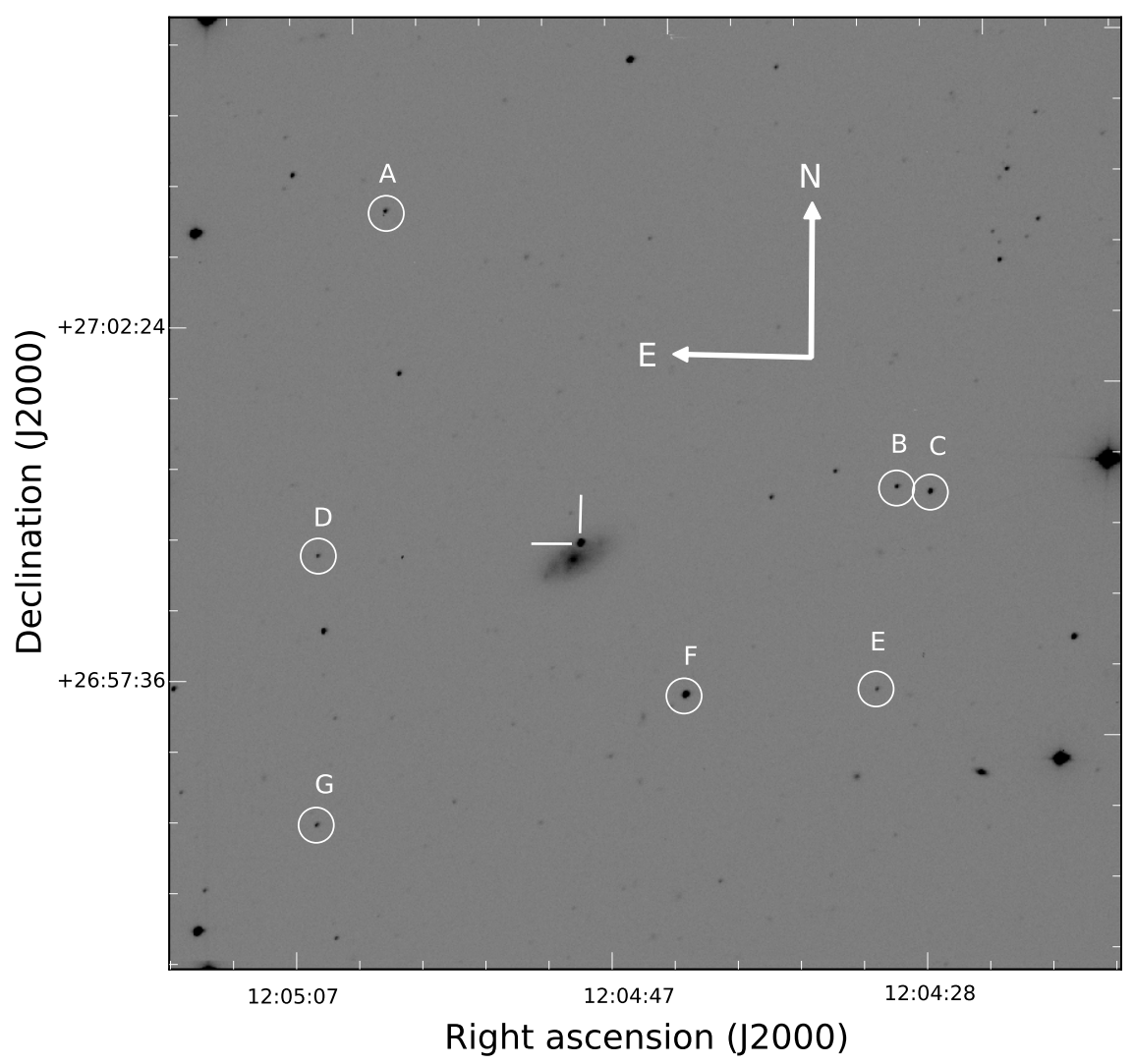

Figure A2. Location of M12045 along with seven local standards used for calibration. The $R$ band image was taken with $104 \mathrm{~cm}$ ST on 15 November, 2014. 
Table A1. Star ID along with co-ordinates and magnitude of secondary standard stars in $B V R I$ bands.

\begin{tabular}{ccccccc}
\hline \hline Star ID & R.A.(h:m:s) & Dec.(d:m:s) & $B(\mathrm{mag})$ & $V(\mathrm{mag})$ & $R(\mathrm{mag})$ & $I(\mathrm{mag})$ \\
\hline A & $12: 05: 04.52$ & $+27: 04: 06.8$ & $17.882 \pm 0.004$ & $17.254 \pm 0.007$ & $16.839 \pm 0.036$ & $16.514 \pm 0.012$ \\
B & $12: 04: 32.39$ & $+27: 00: 47.7$ & $18.288 \pm 0.006$ & $17.481 \pm 0.004$ & $16.882 \pm 0.028$ & $16.463 \pm 0.010$ \\
C & $12: 04: 30.35$ & $+27: 00: 45.6$ & $16.718 \pm 0.002$ & $13.373 \pm 0.003$ & $16.020 \pm 0.036$ & $15.716 \pm 0.007$ \\
D & $12: 05: 07.34$ & $+26: 59: 24.9$ & $18.698 \pm 0.007$ & $17.961 \pm 0.005$ & $17.468 \pm 0.015$ & $17.068 \pm 0.022$ \\
E & $12: 04: 32.88$ & $+26: 58: 02.6$ & $18.435 \pm 0.007$ & $17.935 \pm 0.004$ & $17.532 \pm 0.014$ & $17.183 \pm 0.010$ \\
F & $12: 04: 44.61$ & $+26: 57: 48.9$ & $16.008 \pm 0.002$ & $14.998 \pm 0.003$ & $14.411 \pm 0.023$ & $14.021 \pm 0.010$ \\
G & $12: 05: 06.39$ & $+26: 55: 46.0$ & $18.143 \pm 0.004$ & $17.497 \pm 0.004$ & $17.028 \pm 0.017$ & $16.668 \pm 0.008$ \\
\hline
\end{tabular}

Table A2. Optical photometry of M12045 in BVRI bands.

\begin{tabular}{|c|c|c|c|c|c|c|c|}
\hline Date & $\begin{array}{l}\text { Phase }^{\dagger} \\
\text { (Days) }\end{array}$ & $\begin{array}{c}B \\
(\mathrm{mag})\end{array}$ & $\begin{array}{c}V \\
(\mathrm{mag})\end{array}$ & $\begin{array}{c}R \\
(\mathrm{mag})\end{array}$ & $\begin{array}{c}I \\
(\mathrm{mag})\end{array}$ & $\begin{array}{c}W \\
(\mathrm{mag})\end{array}$ & Telescope \\
\hline 20141028 & 20.87 & - & - & - & - & $14.49 \pm 0.21$ & MASTER \\
\hline 20141114 & 38.24 & - & $15.04 \pm 0.01$ & $14.39 \pm 0.01$ & - & - & $\mathrm{ST}$ \\
\hline 20141115 & 39.25 & $15.07 \pm 0.02$ & $15.12 \pm 0.02$ & $14.38 \pm 0.02$ & - & - & $\mathrm{ST}$ \\
\hline 20141129 & 53.19 & - & $15.28 \pm 0.01$ & - & $14.20 \pm 0.01$ & - & $\mathrm{ST}$ \\
\hline 20141208 & 62.18 & - & $15.40 \pm 0.01$ & $14.83 \pm 0.02$ & $14.92 \pm 0.01$ & - & ST \\
\hline 20141209 & 63.20 & $17.01 \pm 0.01$ & $15.39 \pm 0.01$ & $14.80 \pm 0.01$ & $14.93 \pm 0.00$ & - & ST \\
\hline 20141210 & 64.15 & $17.14 \pm 0.02$ & $15.38 \pm 0.01$ & $14.84 \pm 0.01$ & $14.98 \pm 0.01$ & - & $\mathrm{ST}$ \\
\hline 20141216 & 70.21 & - & $15.48 \pm 0.02$ & $14.93 \pm 0.01$ & $15.05 \pm 0.01$ & - & ST \\
\hline 20141229 & 83.15 & - & $15.66 \pm 0.01$ & $15.15 \pm 0.02$ & $15.30 \pm 0.01$ & - & ST \\
\hline 20150106 & 91.20 & - & $15.72 \pm 0.02$ & $15.24 \pm 0.01$ & $15.36 \pm 0.01$ & - & $\mathrm{ST}$ \\
\hline 20150113 & 97.05 & - & - & - & - & $15.61 \pm 0.12$ & MASTER \\
\hline 20150114 & 98.04 & - & - & - & - & $15.60 \pm 0.06$ & MASTER \\
\hline 20150116 & 100.05 & - & - & - & - & $15.61 \pm 0.03$ & MASTER \\
\hline 20150117 & 101.07 & - & - & - & - & $15.44 \pm 0.06$ & MASTER \\
\hline 20150122 & 106.89 & - & - & - & - & $15.61 \pm 0.19$ & MASTER \\
\hline 20150124 & 108.68 & $17.16 \pm 0.01$ & $16.00 \pm 0.03$ & - & $15.73 \pm 0.02$ & - & $\mathrm{ST}$ \\
\hline 20150130 & 115.10 & $17.23 \pm 0.02$ & - & $15.68 \pm 0.01$ & $15.80 \pm 0.01$ & - & ST \\
\hline 20150131 & 116.18 & $17.34 \pm 0.01$ & $16.14 \pm 0.02$ & $15.68 \pm 0.02$ & $15.86 \pm 0.01$ & - & $\mathrm{ST}$ \\
\hline 20150204 & 120.16 & $17.67 \pm 0.03$ & $16.09 \pm 0.02$ & $15.76 \pm 0.02$ & $15.95 \pm 0.02$ & - & ST \\
\hline 20150205 & 121.06 & $17.71 \pm 0.01$ & $16.19 \pm 0.02$ & $15.75 \pm 0.01$ & - & - & ST \\
\hline 20150216 & 131.82 & - & - & - & - & $15.63 \pm 0.17$ & MASTER \\
\hline 20150225 & 140.90 & - & - & - & - & $16.10 \pm 0.06$ & MASTER \\
\hline 20150322 & 165.90 & $17.74 \pm 0.02$ & $17.19 \pm 0.02$ & $16.44 \pm 0.01$ & $16.32 \pm 0.02$ & - & ST \\
\hline 20150323 & 167.08 & - & $17.00 \pm 0.02$ & $16.46 \pm 0.01$ & $16.74 \pm 0.01$ & - & ST \\
\hline 20150327 & 170.80 & - & - & - & - & $16.28 \pm 0.05$ & MASTER \\
\hline 20150406 & 180.97 & - & $17.26 \pm 0.05$ & $16.67 \pm 0.01$ & $16.95 \pm 0.01$ & - & ST \\
\hline 20150407 & 181.94 & - & $17.31 \pm 0.02$ & $16.67 \pm 0.01$ & $16.90 \pm 0.02$ & - & ST \\
\hline 20150408 & 182.93 & - & - & $16.70 \pm 0.01$ & $16.96 \pm 0.02$ & - & ST \\
\hline 20150409 & 184.07 & - & $17.29 \pm 0.01$ & - & - & - & ST \\
\hline 20150411 & 186.06 & - & - & - & $16.51 \pm 0.02$ & - & ST \\
\hline 20150418 & 192.93 & - & $17.38 \pm 0.02$ & $16.89 \pm 0.02$ & $17.06 \pm 0.02$ & - & $\mathrm{ST}$ \\
\hline 20150421 & 195.92 & - & $17.44 \pm 0.02$ & $16.90 \pm 0.02$ & $16.95 \pm 0.03$ & - & ST \\
\hline 20150424 & 198.97 & - & $17.51 \pm 0.03$ & $16.91 \pm 0.03$ & - & - & ST \\
\hline 20150501 & 205.91 & - & $17.71 \pm 0.04$ & $17.02 \pm 0.01$ & $17.47 \pm 0.03$ & - & ST \\
\hline 20150502 & 206.92 & - & $17.69 \pm 0.02$ & $17.12 \pm 0.02$ & $17.30 \pm 0.04$ & - & ST \\
\hline 20150503 & 207.96 & - & $17.67 \pm 0.02$ & $17.05 \pm 0.01$ & $17.44 \pm 0.02$ & - & ST \\
\hline 20150605 & 240.65 & - & - & - & $17.76 \pm 0.05$ & - & $\mathrm{ST}$ \\
\hline 20150607 & 242.61 & - & - & - & $17.67 \pm 0.02$ & - & $\mathrm{ST}$ \\
\hline 20150610 & 245.65 & $18.96 \pm 0.01$ & $18.33 \pm 0.01$ & $17.62 \pm 0.01$ & $17.82 \pm 0.02$ & - & HCT \\
\hline 20150730 & 295.77 & - & - & - & - & $16.66 \pm 0.06$ & MASTER \\
\hline
\end{tabular}


Table A3. Log of spectroscopic observations.

\begin{tabular}{cccc}
\hline \hline Date & $\begin{array}{c}\text { Phase }^{\dagger} \\
\text { (Days) }\end{array}$ & Grism & $\begin{array}{c}\text { Spectral Range } \\
(\AA)\end{array}$ \\
\hline 20141029 & 20.88 & Gr07,Gr08 & $3800-6840,5800-8350$ \\
20141111 & 34.94 & Gr07,Gr08 & $3800-6840,5800-8350$ \\
20141117 & 40.96 & Gr07,Gr08 & $3800-6840,5800-8350$ \\
20141123 & 46.94 & Gr07,Gr08 & $3800-6840,5800-8350$ \\
20141201 & 54.90 & Gr07,Gr08 & $3800-6840,5800-8350$ \\
20141204 & 57.87 & Gr07,Gr08 & $3800-6840,5800-8350$ \\
20141219 & 72.84 & Gr07,Gr08 & $3800-6840,5800-8350$ \\
20141226 & 79.93 & Gr07,Gr08 & $3800-6840,5800-8350$ \\
20150104 & 88.78 & Gr07,Gr08 & $3800-6840,5800-8350$ \\
20150106 & 90.80 & Gr07,Gr08 & $3800-6840,5800-8350$ \\
20150111 & 95.88 & Gr07,Gr08 & $3800-6840,5800-8350$ \\
20150118 & 102.01 & Gr07,Gr08 & $3800-6840,5800-8350$ \\
20150125 & 109.76 & Gr07,Gr08 & $3800-6840,5800-8350$ \\
20150201 & 116.01 & Gr07,Gr08 & $3800-6840,5800-8350$ \\
20150205 & 120.84 & Gr07,Gr08 & $3800-6840,5800-8350$ \\
20150207 & 122.83 & Gr07,Gr08 & $3800-6840,5800-8350$ \\
20150214 & 129.82 & Gr07,Gr08 & $3800-6840,5800-8350$ \\
20150222 & 137.96 & Gr08 & $5800-8350$ \\
20150303 & 146.75 & Gr08 & $5800-8350$ \\
20150325 & 168.81 & Gr07,Gr08 & $3800-6840,5800-8350$ \\
20150403 & 177.67 & Gr07,Gr08 & $3800-6840,5800-8350$ \\
20150424 & 198.79 & Gr08 & $5800-8350$ \\
20150505 & 209.68 & Gr07,Gr08 & $3800-6840,5800-8350$ \\
20150513 & 217.60 & Gr07,Gr08 & $3800-6840,5800-8350$ \\
20150522 & 226.75 & Gr07,Gr08 & $3800-6840,5800-8350$ \\
20150530 & 234.66 & Gr07 & $3800-6840$ \\
20150611 & 246.67 & Gr07,Gr08 & $3800-6840$ \\
\hline
\end{tabular}

$\dagger \overline{\text { Phase has been calculated with respect to } B_{\max }=2456938.49}$ (JD) 